International Journal of Engineering \& Technology, $7(2.34)(2018) 39-43$
International Journal of Engineering \& Technology
WPC
Website: www.sciencepubco.com/index.php/IJET
Research paper

\title{
Detection And Restoration of Cracked Digitized Paintings and Manuscripts Using Image Processing
}

\author{
Nawafil Abdulwahab Farajalla Ali *, Imad Fakhri Taha Al-Shaikhli, Raini Hasan \\ Department of Computer Science, Information Communication Technology \\ International Islamic University Malaysia, Kuala Lumpur Malaysia \\ *Corresponding author E-mail: nawafil.ali@gmail.com
}

\begin{abstract}
Ancient paintings are cultural heritage that can be preserved via computer aided analysis and processing. These paintings deteriorate due to undesired cracks, which are caused by aging, drying up of painting material, and mechanical factors. These heritages need to be restored to their respective original or near-original states. There are different techniques and methodologies that can be used to conserve and restore the overall quality of these images. The main objective of this study is to analyze techniques and methodologies that have been developed for the detection, classification of small patterns, and restoration of cracks in digitized old painting and manuscripts. The purpose of the developed algorithm is to identify cracks using the thresholding operation, which was the output of the top-hat transform morphology. Afterwards, the breaks, which were wrongly identified as cracks, were separated for utilization in a semi-automatic procedure based on region growth. Finally, both the median filter and weighted median techniques were applied to fill the cracks and enhance image quality.
\end{abstract}

Keywords: Cracks, Detection, Classification, filling, Restoration, Image processing.

\section{Introduction}

Technology has progressed exponentially in the past few decades. Every day, a huge amount of data is generated due to the rapidly increasing size of the digital storage space, as well as the advent of the World Wide Web (WWW). Information could contain images or video sequences, as well as synthetic illustration diagrams, charts, or computer aided graphics [1]. Many institutions, such as museums, libraries, galleries, and archives are digitizing their collections to simplify public access. Creating and maintaining the best possible environment for storing or displaying artifacts help prevent damages and increase longevity [2]. It is the prime duty of the custodians of the museums, libraries, galleries, and archives to preserve and conserve their collection, not only in its physical forms, but also its digital counterparts [3].

Digital storage allows the images to be manipulated without defacing the original. Collections of paintings saved in museums were from centuries ago, which necessitates its protection and restoration so that it remains in excellent shape indefinitely. Cracking is a common problem in old paintings. A crack appears on the paintings' surfaces when pressures develop within or on it due to various factors, which culminates in the materials' breaking $[4,5]$.

Several paintings, especially old ones, suffer from breaks in its substrate, paint, or varnish. These are commonly called cracks or craquelure. They are mainly caused by aging, drying, and mechanical factors. Age cracks are formed due to the non-uniform reduction in the woodpanel support or canvas of the painting, which induce a pressure on the painting's surface [6]. Drying cracks affect the evaporation of the volatile paint's composition and the subsequent contraction of the paint. Finally, mechanical actions are induced by painting deformations via external factors, such as projection physical damage impacts [7, 8].

Image processing techniques can be used to develop algorithms that can detect and classify cracks on old digitized paintings and manuscripts [9]

This paper is organized in the following manner. Sections 2 talk about related work of detection and restoration techniques. Section 3 details the proposed work, while section 4 discusses crack detection techniques. And section 5 explains the detection and classification of thin scratches that could be misidentified as cracks. Section 6 discusses crack filling, while section 7 detail the results. Section 8 conclude the work and outline a few recommendations.

\section{Related Work}

Restoration of digitized paintings is one of the major concern of this paper. As the appearance of cracks on paintings deteriorates it, the perceived image quality will deteriorate as well. There are many techniques and methodologies that can be used to detect and restore cracks in digitized old paintings and manuscripts. The selection of specific techniques based on our requirements is therefore crucial.

Ioannis \& Giakoumis et. al [10] proposed an integrated methodology to detect cracks and remove them from old digitized paintings. First, the threshold technique was used to detect cracks on images post-application of the top-hat transform morphology. Thin dark strokes created from the artists' brush were removed using a semi-automatic procedure based on the region growing method or median radial basis function neural network on the hue and saturation data. Finally, the cracks were interpolated using a controlled anisotropic diffusion or order statistics filters. This 
methodology can be used for the virtual restoration of deteriorated images, and was proven to be quite effective vis-à-vis cultural paintings of high artistic value. The integrated methodology could not detect cracks located on very dark image regions and cracks that cross the border between regions of different colors.

Sachin et. al [11] reported a technique that can be used to monitor and interpolate cracks in old heritage digitized paintings. They applied the top-hat transformation algorithm to detect cracks and identify misclassified cracks by separating them from the entire image using a semi-automatic approach based on the growth area technique, where a suitable technique that modify the order statistics was performed. The results of the proposed technique confirmed its viability in distinguishing the different transformation morphologies.

Schirripa \& et. al [4] al proposed a new full automatic approach for crack detection and restoration of historical digitized painting. First, the cracks were detected using the top-hat opening and closing operator transformation method, followed by the separation of brush scratches created from the artist brush being misclassified as cracks on the basis of the Hue and saturation values technique[12] Finally, the texture synthesis algorithm was used to fill the cracks. The results indicated that the full automatic methodology can efficiently extract the cracks and enhance image quality by filling the cracks.

Shilpa \& Nisha [9] proposed an algorithm for crack detection and restoration in old historical digitized paintings. They applied a region growing algorithm on the threshold technique, which is an output of the top-hat transform technique that extract information from the background of an image, and use them to distinguish crack patterns from the strokes of a brush that have been mistakenly identified as cracks \{Barni, 2005 \#49\} Furthermore, the anisotropic diffusion technique and order statistics filtering were used to fill cracks and restore the digital images. The results confirmed the viability of this method for the classification of crack patterns from images via the combination of two effective morphology techniques.

Shruti \& Sahoo [13] proposed a technique to detect, restore, and identify cracks in old damaged digitized paintings. A low pass filter was used to locate the cracks. Supervised and unsupervised classification methods were then introduced. Four different methods were used to classify cracks from dark black brush strokes prior to it being filled, which were Back propagation, k-nearest neighborhood, k-mean clustering, and Fuzzy c mean clustering. The results indicated that the fuzzy c-means classifier is the best in the case of many paintings, but it selects random cluster center initially so that the classes could be interchanged in multiple runs. Entropy-based clustering also reported excellent results; it reported lesser brush strokes than fuzzy c, which means that clustering also resulted in a lesser number of crack pixels being misclassified as brush strokes.

Mohammed et. al [14] proposed an automated method for detecting and eliminating cracks in old deteriorated digitized paintings. They used the top-hat transform morphology to locate the cracks. The Gaussian Classifier technique was used to separate smal pattern areas from the cracks. Then, a new filter called Modified Trimmed Mean Filter (MTMF) and the weighted median filter were used to fill the cracks. The results indicated that the intensity of the crack pixels in these areas were very close to the intensity of the surrounding pixels, rendering this algorithm ineffective in detecting cracks in very dark images.

Anubhav et. al [15] proposed an integrated algorithm composed of three applications for image processing. The first step was crack detection, where they applied the thresholding technique to generate the morphological top-hat transform to distinguish crack patterns from the background of the image. Then, thin dark strokes of brushes misclassified as cracks were removed using the hit-andmiss cleaning algorithm Finally, crack filling or restoration was carried out using the median and weighted median filters. The image quality was excellent due to the usage of different tech- niques to detect and remove cracks from images and fill the cracks so that the images can be enhanced.

Pratap \& Mandal [16] introduced an integrated method for detecting and eliminating cracks in old heritage digitized paintings. They applied the top-hat and bottom-hat transformations based on the structuring element and its shape to probe/interact with a given image. Then, a semi-automatic technique was used to extract the cracks using the hue and saturation technique to separate the thin strokes of the artists' brush from the cracks that was mistakenly identified as cracks. The cracks were then filled using two approaches; inpainting, which is mostly pixel-based, and the pixelbase and patch-based texture synthesis[17]. Both looked for and copied the pixels that were most similar to its respective local neighborhoods as its synthetic texture base. The results showed the declination of image quality, which necessitates the usage of the apply detection and separation technique to enhance the image's quality.

Kaushik et. al [18] introduced a method to develop a semiautomatic crack detection system that analyzes the concrete surface and detect cracks from digitized paintings. The crack detection procedure used the morphological bottom-hat transformation to separate the pattern from the background of the image [19]. Then, the crack was filled by applying the order static median filter based on the information of the neighborhood pixels. The results confirmed significant problems in detecting cracks based on morphological transformation.

After studying various techniques of old painting restoration. There are many problems in existing image restoration techniques. Making fragments of old painting is an arduous task, at best. Morphological algorithm only detects the cracks and missing areas in old paintings' surface. To overcome these limitations, a new algorithm that can detect and remove cracks is needed.

\section{Proposed method and implementation}

The proposed method implements a crack detection and restoration techniques, which consists of three main steps that can be combined into stages, as per Fig. 1

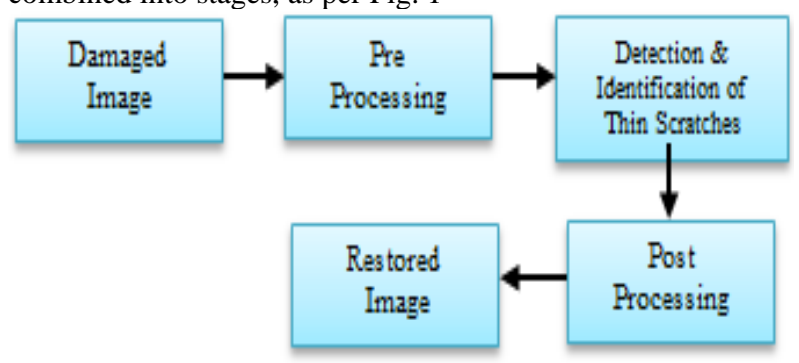

Fig. 1: The Main Structure of the proposed method

The concept used here is image processing techniques for the analysis, preservation, and restoration of artworks. The main objective of the proposed system is to detect and restore cracks and remove scratches and other artifacts from digitized painting and manuscripts. Such methods use information obtained over several adjacent frames. The block diagram of the proposed work is shown in Fig. 1, while the approach used for crack detection \& restoration is detailed below:

\subsection{Damaged image}

The images were acquired from various databases, and then converted to compressed file formats with low storage requirements.

\subsection{Pre-processing image}

The stage involves colored images. These color images need to be converted into common color formats, such as a grey scale colored 
image. The threshold morphology technique then needs to be used to create an image mask.

\subsection{Detection and classification of thin scratches}

This stage of painting classification is divided into two steps: detection and classification of the thin scratches that were misidentified as cracks. The current method uses both feature extraction and color information.

\subsection{Post-processing image}

After detecting and separating cracks, the cracks were filled with its surrounding pixels' color.

\subsection{Restored image}

This module will produce the restored image post crack filling.

\section{Crack detection}

Crack detection is similar to crack recognition, which is also known in literature as structure extraction. Therefore, crack detection is a method of extracting information from images based on the application of different/specific algorithms. Normally, cracks have a local intensity minima/local intensity maxima, which make them dark cracks (low luminance) or light cracks (high luminance). It is therefore reasoned that crack detection techniques applied on the luminance elements should be able to identify these minima from images.

\subsection{Top-hat transform morphology}

This approach is illustrated for crack detection procedures that separate cracks from the images:

$y(x)=f(x)-f$ n $B(x)$

where $f n B(x)$ defined as the opening of the function of $f(x)$, with a structuring set:

n $\mathrm{B}=\mathrm{B}$ Ө B $\Theta$ B----B (n times)

In equation (2), $\Theta$ denotes the defined dilation operation. Once equation (2) is evaluated, it produces a final structuring set, and is subsequently utilized in the opening operation of equation (1). The low-pass nonlinear filter, identified as the opening of the function $f(x)$, eliminates all local maxima of the structuring element that are not good fits. This process produces only the local maxima, which is recognized as cracks without the background of the digital image, since cracks are identified as local intensity minima structure instead of the local intensity maxima. Better results can be obtained from the opposite luminance image when the top-hat transform morphology filter is used. The output of the crackdetection procedure can be controlled via user intervention by controlling outputs and selecting appropriate values for the following quantities:

- Select the shape and the size of structuring element.

- $\quad$ Select the structuring element size which it $3 \times 3$.

- The number of times is performed in equation no. (2).

The resultant structuring element size is affected by these numbers. They need to be selected based on the broadness of the cracks being detected.

\subsection{Threshold operation}

The threshold operation is a method that can be used to extract crack information from the background of images and the number of pixels extracted from digital images. The use of the threshold method on the images would increase the value of the number of crack pixels. Therefore, the images with cracks in its dark region will probably not be properly processed, since its local minima condition can remain undetected due to the limitation of this method. The false identification of cracks is therefore an obvious problem in this approach. The proposed algorithm is detailed as follows:

Input: Original Image

Output: Binary cracked Image

Step 1: Load the cracked image

Step 1: Convert the colored image to Grayscale image

Step 1: Take the size of image $\mathrm{m} \times \mathrm{n}$ (the mask)

Step 1: Run the first mask on the image

Step 1: Grayscale dilatation of step 2

Step 1: Define Threshold

Step 1: Extract binary mask using define Threshold

Step 1: Apply median filter to the cracked region

Step 1: Add the output of both masks

Step 1: Display the output

\section{Detection and identification of thin scratch- es which misclassify as cracks}

Many old paintings suffer from breaks, which lowers its quality. Breaks are called cracks as they contain similar luminance and structure features with that of cracks. The possibility of misidentifying cracks as breaks, and vice versa, is possible when using the top-hat transform morphology technique. These cracks must be separated from the original cracks prior to the application of the crack filling method. Its procedural outlined is detailed in the following subsection.

\subsection{A semi-automatic crack separation method}

This procedure separate patterns from cracks. They apply a region growing algorithm on the threshold operation method, which is the output of the top-hat transform morphology. The cracks originate from a pixel seed. The user selects the pixels in an interactive manner. Minimally, one seed should be selected for each crack element. The user can also apply this method on the breaks.

The region growth mechanism is recursively checked for misclassified pixels containing value 1 in the 8-neighborhood of each crack pixel. Until the processes reach the final phase of the image and find the pixels in the binary digital image, it goes along the cracks that do not belong to the 8-connected to breaks.

Cracks are mainly characterized via the application of the threshold technique to uniform at the greyscale level. The cracks can be detected using both techniques. However, the cracks are regarded as local intensity minima (low luminance) relative to its background. New cracked pixels are allocated when the system identify pixels that belong to a crack if their grey levels are false within a given range and do not change significantly from those of the pixels already classified as belonging to the cracks. Fig. 2 shows the tracking procedure of the three iterations. The starting crack point is A, which is defined by the user. Pixel A is measured by identifying the 8-neighborhood (from pixels B1-B5). For each pixel $\mathrm{Bi}$ of the neighborhood, the system would test the following conditions:

$|f(A)-f(B i)|<=T$

$\mathrm{f}(\mathrm{Bi}) \quad \mathrm{\epsilon}[\mathrm{T} 1, \mathrm{~T} 2]$ 
Where $\mathrm{f}(\mathrm{Bi})$ is identified as a greyscale level, which is $\mathrm{Bi}$, while $\mathrm{T}$, $\mathrm{T} 1$ and $\mathrm{T} 2$ are mainly adaptive as threshold operation, calculated on the basis of crack pixels previously classified as such.

As shown in Fig. 2, only pixels are seen in the system. Pixels B1, $\mathrm{B} 2$, and $\mathrm{B} 3$ belong to the crack. The method tracks the pixels up till those that are close to pixels B1 - B3 and $\mathrm{C} 1$ - C6. The conditions of both equation no. 3 and no. 4 are examined by the system. The validity of condition no. 3 is then confirmed for each pair of pixels belonging to the same 8-neighborhood window. Therefore, pixel $\mathrm{Ci}$ is supposed to fit in the crack area if it supports condition no. 4. Condition no. 3 is validated in the case of pixel $\mathrm{Bj}$ in the neighborhood window of $\mathrm{Ci}$ (in Fig. 2), and only pixel C5 is identified as a crack pixel). The previous procedure iterates until the system discontinues to obtain pixels with suitable structures. When cracks traverse on fronts such as $(\{\mathrm{B} 1, \mathrm{~B} 2, \mathrm{~B} 3\}$ at iteration $1,\{C 5\}$ will be at iteration 2 , and $\{\mathrm{D} 2, \mathrm{D} 3\}$ at iteration 3$)$.

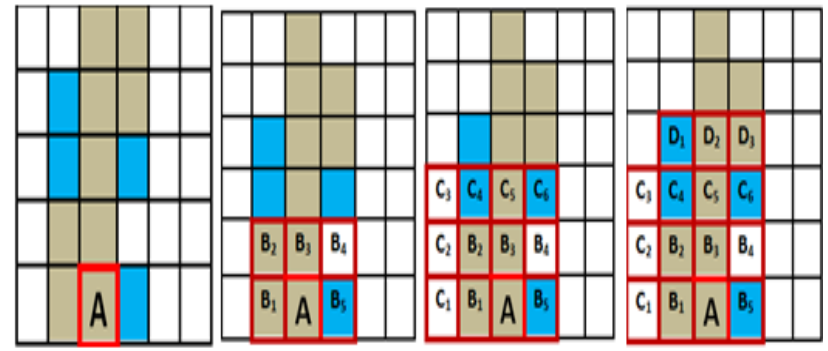

Fig. 2: Shows the three tracking of pixel A

\section{Crack filling}

After identifying and classifying the cracks, the pre- processing stage involves restoring the image using local image information that uses information from neighboring pixels to fill the cracks. The median filter and weighted median filter techniques can be used for this purpose, and the visual inspection of the results was evaluated after the implementation of the crack filling methods, as shown below.

The neighborhood pixels can be used to interpolate and effectively restore the cracks. The filter window center method is applied on the crack pixels. When the filter window size is sufficiently large (3x3), then the crack pixels within the window is disallowed, as its outliers are smaller. Therefore, the value of one neighboring pixel that do not belong to the crack pixels will be allocated in the crack pixel. These filters can be used for the following purpose:

\subsection{Order statistics filters for interpolation}

An important way to interpolate the cracks is by utilizing the median filter. The filters will be selectively implemented on the crack pixel. For example, only the crack pixels are assigned to the core of the window of the filter traverses. Hence, the crack pixels allocated within the window of the filter will be assigned an outline, then kept aside based on the size of the filter window being passably bigger. This means that any of the neighborhood non-cracked pixels will be selected. There are various filters that can be used for this purpose.

\subsubsection{Median filter}

$y i=\operatorname{med}(x i-v, \ldots \ldots x i, \ldots \ldots . . x i+v)$

\subsubsection{Recursive Median filter}

$y i=\operatorname{med}(y i-v, \ldots y i-1, x i, \ldots \ldots . . x i+v)$

Where yi-v,...., yi-1 are the initially calculated median resultan samples for both filters median and recursive median utilization The size of the filter window (which is only produced in rectangular window shape) should correspond to $50 \%$ larger than the thickest crack visible on the cracked image. This is very important, as it confirms the filter outcome that has been selected to be the value of the incorrect crack pixel. The cracks that will not be passably filled will result in all of the windows having small sizes, while the cracks that are much thinner than the windows with large uniform windows will be acquired, which reduces image quality.

\subsubsection{Weighted Median filter}

$\mathrm{yi}=\operatorname{med}(\mathrm{w}-\Theta x \mathrm{x}-\mathrm{v}, \quad, \mathrm{wv} \Theta \mathrm{xi}+\mathrm{v})$

Where $\diamond$ denotes $\mathrm{x}$, w times. This sort of filter is smaller, which is $\sim 30 \%$ wider than the thickest crack seen on the image. It can also be used as a filter due to the probability of a color value corresponding to a crack. The result of having small weights for the pixels can be restricted within the window size, in some cases to larger ones than the pixels. The modified trimmed mean (MTM) filter usually deal with filter window and avoid taking the variable of samples it is located in. However, the average of the remaining pixels is known to be smaller from the local median.

\section{Results and discussion}

The experiment was performed on some digitized old paintings images by applying different techniques that identifies the missing/damaged pixel of the image regions by filling in the crack information using the neighboring pixel method. Crack classification procedure was conducted using paints to repair the deterioration. The experiment was carried out in MATLAB.

Fig. 3 shows the original colored images that suffer from cracks that need to be restored and displayed on GUI:

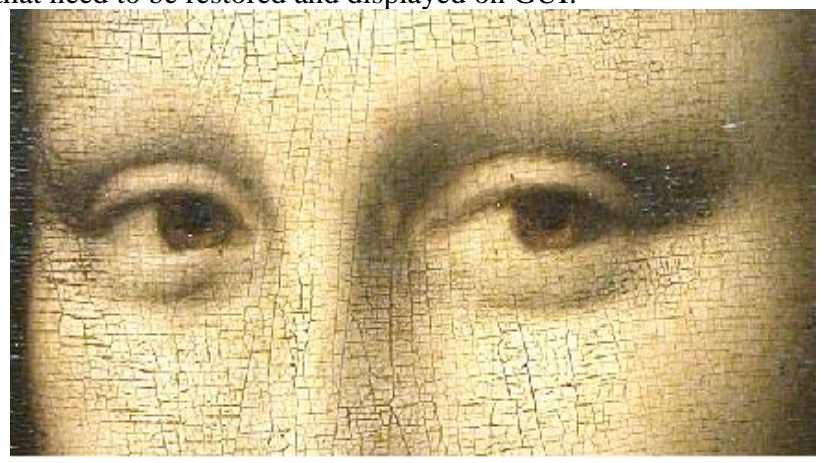

Fig. 3: Original cracked image

In Fig. 4 the top-hat transform morphology, which separates the cracks from the rest of the image, was utilized to produce a grayscale level resultant image that is the large greyscale level value where the pixels are on genuine cracks or the crack act as structures for displaying it on the GUI:

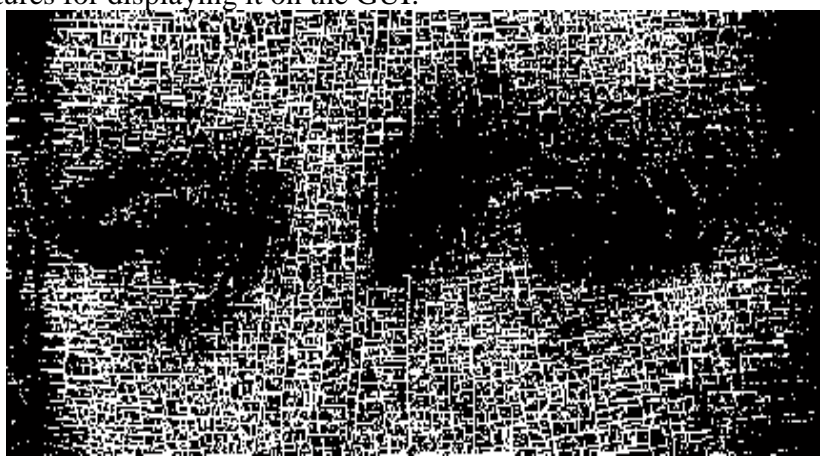

Fig. 4:Cracked Detection

The binary output image is the result of the application of the local intensity minima (low luminance) module in cracked image. The following experiment used these parameters: 
- The squared shape presents the structuring element.

- $3 \times 3$ sizes produce structuring element.

- 2 dilations process.

\section{Conclusion and future work}

In this research, the detection and restoration of cracks in old digitized painting and manuscripts was reported. Binary images were acquired by applying the threshold operation, which is the output of the top-hat transform morphology technique. Then, a thin scratch, which has been misidentified as a crack, was obtained via a semiautomatic crack separation method. The two methodologies that has been used to fill the crack were the median filter and weighted median filter techniques. MATLAB was used to build and test the complete system. Different techniques and strategies were used to detect and fill old digitized paintings. There are certain aspects of the proposed methodology that could use some improvements, such as the very dark areas in certain parts of the image and the techniques used in the crack-detection stage not being very efficient for detecting cracks due to the intensity of the surrounding pixels being very close to the intensity of the crack pixels. Future works could focus on:

- Applying the feature extraction technique to classify the type and size of crack, and then using a restoration method on the old digitized artistic image and manuscript.

- Artificial intelligence approach will be utilized to analyze and identify patterns from the background of an image.

- Select a low threshold transform value to operate crack detection algorithms locally and on its surrounding region(s).

\section{Acknowledgements}

This material is based upon work supported by the IIUM under Grant No. RIGS16-366-0530

\section{References}

[1] I. G. a. I. Pitas, "Digital Restoration Of Painting Cracks " IEEE Signal processing magazine, p. 4, 2001.

[2] I. Giakoumis, N. Nikolaidis, and I. Pitas, "Digital image processing techniques for the detection and removal of cracks in digitized paintings," IEEE Transactions on Image Processing, vol. 15, pp. 178-188, 2006.

[3] F. Abas and K. Martinez, "Craquelure analysis for content-based retrieval," in Digital Signal Processing, 2002. DSP 2002. 2002 14th International Conference on, 2002, pp. 111-114.

[4] b. Qin Zoua, c, Yu Caoc, Qingquan Lib,d, Qingzhou Maob,d, Song Wangc "Automatic crack detection from pavement images," p. 11, 2011.

[5] M. Barni, A. Pelagotti, and A. Piva, "Image processing for the analysis and conservation of paintings: opportunities and challenges," IEEE Signal processing magazine, vol. 22, pp. 141-144, 2005.

[6] D. S. R. Y. Sateesh.V1, "Implementation Of Detection And Removal Of Cracks In Digitized Paintings Using Dip Techniques," Global Journal of Advanced Engineering Technologies, 2012.

[7] V. K. Abhilekh Gupta, Abhinav Gupta and M. C. Srivastava, "Image Processing Methods for the Restoration of Digitized Paintings," Thammasat Int. J. Sc. Tec, vol. 13, p. 7, 2008.

[8] R. K. G. P. M. P. Parsai2, "Restoration of Digitized Image of Cracked Paintings -A Review " International Journal for Scientific Research \& Development, vol. 3, p. 3, 2015.

[9] S. A. N. SHARMA2, "Inpainting Approach To Repair Cracked Images " International Journal of Application or Innovation in Engineering \& Management vol. 1, p. 7, 2012.

[10] N. Nikolaidis and I. Pitas, "Digital image processing in painting restoration and archiving," in Image Processing, 2001. Proceedings. 2001 International Conference on, 2001, pp. 586-589.

[11] S. V. S. a. M. A. R. Mahajan2, "Cracks Inspection and Interpolation in Digitized Artistic Picture using Image Processing Approach " In- ternational Journal of Recent Trends in Engineering, vol. 1, p. 3, 2009.

[12] V. V. K. Vidya V. Khandare1, "Digital Restoration of Cracks Based on Image Processing " International Research Journal of Engineering and Technology vol. 2, p. 4, 2015.

[13] G. S. Shruti Garg1, "Classification of Cracks and Brush Strokes in Old Digital Paintings " Int. J. of Recent Trends in Engineering \& Technology, , vol. 11, p. 10, 2014.

[14] N. A. W. F. P. Mohammed AbdALLA A. ElmaleehP1P, Amin Babikr A/Nabi MustafaP2 "Detection and Removal of Cracks in Digitized Paintings via Digital Image Processing " International Journal of Innovative Science, Engineering \& Technology, vol. 1, p 8, 2014.

[15] E. B. S. Sankarasrinivasana, * , K. Karthika, U. Chandrasekarb and Rishi Guptac "Health Monitoring of Civil Structures with Integrated UAV and Image Processing System," Procedia Computer Science p. 8, 2015.

[16] N. K. Deepika Pagrotra1, "A Review Paper on Crack Detection and Restoration of Old Painting," International Journal of Science and Research vol. 4, p. 3, 2015.

[17] L. Z. Cui Fang 1, and Yao Li 3 "Images Crack Detection Technology based on Improved K-means Algorithm " JOURNAL OF MULTIMEDIA, vol. 9, p. 7, 2014.

[18] K. B. a. S. K. Bandyopadhyay, "Digital Image Processing Techniques For The Detection And Removal Of Cracks In Digitized Paintings Or Concrete Rtructure," International Journal of Current Research, vol. 8, p. 6, 2016.

[19] Bruno Cornelisa, b., Ann Doomsa,b, Jan Cornelisa, Frederik Leenc and Peter Schelken Digital Painting Analysis, At The Cross Section Of Engineering, Mathematics And Culture" 19th European Signal Processing Conference 2076 - 1465, 2011. 